Binary Misclassification and Identification in Regression Models

By: Martijn van Hasselt, Christopher R. Bollinger

Van Hasselt, M., \& Bollinger, C. R. (2012). Binary misclassification and identification in regression models. Economics Letters, 115(1), 81-84. DOI: 10.1016/j.econlet.2011.11.031.

Made available courtesy of Elsevier: http://dx.doi.org/10.1016/j.econlet.2011.11.031

***(C) Elsevier. Reprinted with permission. No further reproduction is authorized without written permission from Elsevier. This version of the document is not the version of record. Figures and/or pictures may be missing from this format of the document. $* * *$

This is the author's version of a work that was accepted for publication in Economics Letters. Changes resulting from the publishing process, such as peer review, editing, corrections, structural formatting, and other quality control mechanisms may not be reflected in this document. Changes may have been made to this work since it was submitted for publication. A definitive version was subsequently published in Economics Letters, Vol. 115, Issue 1, (2012) DOI: 10.1016/j.econlet.2011.11.031

\begin{abstract}
:
We study a regression model with a binary explanatory variable that is subject to misclassification errors. The regression coefficient is then only partially identified. We derive several results that relate different assumptions about the misclassification probabilities and the conditional variances to the size of the identified set.
\end{abstract}

Keywords: Misclassification error | Binary regressors | Partial identification | Homoscedasticity

Article:

1. Introduction

In this note, we study a regression model with a binary explanatory variable that may be misclassified. That is, there is some nonzero probability of observing a "false positive" or a "false negative". For example, such errors can be simple data coding errors, or result from the underreporting of certain behaviors (e.g., illicit drug use) in surveys. In general, the regression parameter is not identified in the presence of misclassification, and different sets of identifying assumptions have been investigated. Chen et al., 2008a and Chen et al., 2008b, Mahajan (2006) and Lewbel (2007) present sufficient conditions for identification. Alternatively, Klepper (1988), Manski (1990), Bollinger (1996), and Deng and Hu (2009) take a partial identification approach and derive bounds of the identified set that are nonparametrically identified under weak assumptions. This note adds to this literature by presenting several new partial identification results. Specifically, we demonstrate to what extent homoscedasticity and restrictions on the 
misclassification probabilities shrink the size of the identified set. We present the model, assumptions and main results in Section 2. We provide a brief discussion in Section 3. All proofs are collected in the Appendix.

2. Main results

The regression model for the outcome variable $Y_{i}$ is given by

equation(1)

$Y_{i}=\alpha+\beta Z_{i}+U_{i}, \quad E\left(U_{i} \mid Z_{i}\right)=0, \quad \sigma_{U}^{2}=E\left(U_{i}^{2}\right)<\infty$,

where $U_{i}$ is an unobserved error term. The regressor $Z_{i} \in\{0,1\}$ is binary, with $\operatorname{Pr}\left\{\mathrm{Z}_{\mathrm{i}}=1\right\}=\pi$ and $\pi \in(0,1)$. Note that linearity of the model is not restrictive since $\mathrm{Z}_{\mathrm{i}}$ is binary. The econometrician does not observe $\mathrm{Z}_{\mathrm{i}}$, but a binary variable $\mathrm{X}_{\mathrm{i}}$, which is a potentially misclassified version of $\mathrm{Z}_{\mathrm{i}}$. Specifically, we assume the following.

Assumption 2.1.

$\operatorname{Pr}\left\{\mathrm{X}_{\mathrm{i}}=1 \mid \mathrm{Z}_{\mathrm{i}}, \mathrm{Y}_{\mathrm{i}}\right\}=(1-\mathrm{q}) \mathrm{Z}_{\mathrm{i}}+\mathrm{p}\left(1-\mathrm{Z}_{\mathrm{i}}\right)$.

\section{Assumption 2.2.}

$\mathrm{p}+\mathrm{q}<1$.

Assumption 2.1 introduces the misclassification probabilities $\mathrm{p}$ and q, and states that $\mathrm{X}_{\mathrm{i}}$ is conditionally independent of the outcome $\mathrm{Y}_{\mathrm{i}}$; hence, the misclassification error contains no information about $\mathrm{Y}_{\mathrm{i}}$, or vice versa. ${ }^{1}$ In some applications, however, this assumption may be untenable (e.g., Kreider and Pepper, 2007). Assumption 2.2 ensures that the covariance between $Z_{i}$ and $X_{i}$ is positive, so that $X_{i}$ is a better predictor of $Z_{i}$ than a purely random guess.

The mean, variance and covariance of $\left(\mathrm{X}_{\mathrm{i}}, \mathrm{Y}_{\mathrm{i}}\right)$ are given by ${ }^{2}$

$\mu_{X}=(1-\pi) p+\pi(1-q)$

equation(2)

$\mu_{Y}=\alpha+\beta \pi$,

$\sigma_{X Y}=\beta \pi(1-\pi)(1-p-q)$,

$\sigma_{Y}^{2}=\beta^{2} \pi(1-\pi)+\sigma_{U}^{2}$.

These moments of $\left(\mathrm{X}_{\mathrm{i}}, \mathrm{Y}_{\mathrm{i}}\right)$ are nonparametrically identified. If $\sigma_{\mathrm{XY}}=0$, then it follows from (2) that $\left(\boldsymbol{\alpha}, \boldsymbol{\beta}, \boldsymbol{\sigma}_{U}^{2}\right)$ is identified. To avoid this trivial case, we impose the following:

\section{Assumption 2.3.}


$\sigma_{\mathrm{XY}}>0$.

Bollinger (1996, Theorem 1) shows that

under Assumption 2.1, Assumption 2.2 and Assumption 2.3:

equation(3)

$$
\begin{gathered}
\frac{\sigma_{X Y}}{\sigma_{X}^{2}} \leq \beta \leq \max \left\{\mu_{X} \frac{\sigma_{X Y}}{\sigma_{X}^{2}}+\left(1-\mu_{X}\right) \frac{\sigma_{Y}^{2}}{\sigma_{X Y}},\right. \\
\left.\left(1-\mu_{X}\right) \frac{\sigma_{X Y}}{\sigma_{X}^{2}}+\mu_{X} \frac{\sigma_{Y}^{2}}{\sigma_{X Y}}\right\} .
\end{gathered}
$$

The first and second bounds on the right-hand side apply for $\mu_{X} \leq \frac{1}{2}$ and $\mu_{X}>\frac{1}{2}$, respectively. Let $\sigma_{j}^{2} \equiv \operatorname{Var}\left(Y_{i} \mid X_{i}=j\right)$ for $\mathrm{j}=0,1$ be the conditional variance of $Y_{\mathrm{i}}$ given $\mathrm{X}_{\mathrm{i}}$. Our first result shows that the bounds can be tightened if the regression error $U_{i}$ is homoscedastic:

\section{Lemma 1.}

If Assumption 2.1, Assumption 2.2 and Assumption 2.3 hold and $E\left(U_{i}^{2} \mid Z_{i}\right)=E\left(U_{i}^{2}\right)=\sigma_{U}^{2}$, then

$$
\begin{gathered}
\frac{\sigma_{X Y}}{\sigma_{X}^{2}} \leq \beta \leq \max \left\{\left(1-\mu_{X}\right) \frac{\sigma_{X Y}}{\sigma_{X}^{2}}+\mu_{X} \frac{\sigma_{1}^{2}\left(1-\mu_{X}\right)^{2}}{\sigma_{X Y}},\right. \\
\left.\mu_{X} \frac{\sigma_{X Y}}{\sigma_{X}^{2}}+\left(1-\mu_{X}\right) \frac{\sigma_{0}^{2} \mu_{X}^{2}}{\sigma_{X Y}}\right\} .
\end{gathered}
$$

The first and second bound on the right-hand side apply for $\mu_{X} \leq \frac{1}{2}$ and $\mu_{X}>\frac{1}{2}$, respectively. The upper bound is sharper than the one in (3).

By comparing the upper bounds of Lemma 1 and Eq. (3) it is easy to see when homoscedasticity is effective in reducing the size of the identified set. For example, suppose that $\mu_{X}<\frac{1}{2}$ and consider increasing values of $\sigma_{0}^{2}$; this leads to a higher value of $\sigma_{Y}^{2}$, which in turn increases the bound in (3). The bound in Lemma 1, however, is unaffected. Intuitively, when $\mu_{X}<\frac{1}{2}$ it is more likely that $\mathrm{X}_{\mathrm{i}}=0$. At the same time a large value of $\boldsymbol{\sigma}_{0}^{2}$ implies substantial variation in the outcome when $X_{i}=0$, making it 'harder' to identify $\beta$. The homoscedasticity assumption is then more valuable in terms of reducing the (absolute and relative) size of the identified set.

The following results consider the effect of various assumptions about $\mathrm{p}$ and $\mathrm{q}$ on the bounds for $\beta$. Lemma 2 complements Theorem 2 in Bollinger (1996):

\section{Lemma 2.}


Suppose that Assumption 2.1, Assumption 2.2 and Assumption 2.3 hold. If $\mathrm{q}=0$ and $\mathrm{p}>0$, then

$\frac{\sigma_{X Y}}{\sigma_{X}^{2}} \leq \beta \leq \mu_{X} \frac{\sigma_{X Y}}{\sigma_{X}^{2}}+\left(1-\mu_{X}\right) \frac{\sigma_{Y}^{2}}{\sigma_{X Y}}$.

If $\mathrm{p}=0$ and $\mathrm{q}>0$, then

$\frac{\sigma_{X Y}}{\sigma_{X}^{2}} \leq \beta \leq\left(1-\mu_{X}\right) \frac{\sigma_{X Y}}{\sigma_{X}^{2}}+\mu_{X} \frac{\sigma_{Y}^{2}}{\sigma_{X Y}}$.

In some cases the restriction that either $\mathrm{p}$ or $\mathrm{q}$ is equal to zero, may be reasonable. For example, individuals are likely to underreport the use of illegal drugs $(q>0)$, but it is highly implausible that a non-user reports actual use $(p=0)$. The upper bounds in Lemma 2 are again sharper than the one in (3). For example, when $\mu_{\boldsymbol{X}}>\frac{1}{2}$ the additional assumption that $\mathrm{q}=0$ reduces the upper bound by an amount

$$
\begin{aligned}
& \left(1-\mu_{X}\right) \frac{\sigma_{X Y}}{\sigma_{X}^{2}}+\mu_{X} \frac{\sigma_{Y}^{2}}{\sigma_{X Y}}-\left(\mu_{X} \frac{\sigma_{X Y}}{\sigma_{X}^{2}}+\left(1-\mu_{X}\right) \frac{\sigma_{Y}^{2}}{\sigma_{X Y}}\right) \\
& =\left(2 \mu_{X}-1\right)\left(\frac{\sigma_{Y}^{2}}{\sigma_{X Y}}-\frac{\sigma_{X Y}}{\sigma_{X}^{2}}\right) \\
& >0 .
\end{aligned}
$$

Next, suppose that false positives are at least as likely as false negatives $(q \leq p)$, or vice versa $(p \leq q)$. Let $\rho_{X Y}$ denote the correlation coefficient. The identified set for $\beta$ then takes the following form:

\section{Lemma 3.}

Suppose that Assumption 2.1, Assumption 2.2 and Assumption 2.3 hold. If $\mathrm{q} \leq \mathrm{p}$, then

$$
\begin{gathered}
\frac{\sigma_{X Y}}{\sigma_{X}^{2}} \leq \beta \leq \max \left\{\mu_{X} \frac{\sigma_{X Y}}{\sigma_{X}^{2}}+\left(1-\mu_{X}\right) \frac{\sigma_{Y}^{2}}{\sigma_{X Y}},\right. \\
\left.\frac{\sigma_{Y}^{2}}{\sigma_{X Y}} \sqrt{1-4 \sigma_{X}^{2}\left(1-\rho_{X Y}^{2}\right)}\right\} .
\end{gathered}
$$

Conversely, if $\mathrm{p} \leq \mathrm{q}$, then

$$
\begin{gathered}
\frac{\sigma_{X Y}}{\sigma_{X}^{2}} \leq \beta \leq \max \left\{\frac{\sigma_{Y}^{2}}{\sigma_{X Y}} \sqrt{1-4 \sigma_{X}^{2}\left(1-\rho_{X Y}^{2}\right)},\right. \\
\left.\left(1-\mu_{X}\right) \frac{\sigma_{X Y}}{\sigma_{X}^{2}}+\mu_{X} \frac{\sigma_{Y}^{2}}{\sigma_{X Y}}\right\}
\end{gathered}
$$


the first and second bounds on the right-hand side apply for $\mu_{X} \leq \frac{1}{2}$ and $\mu_{X}>\frac{1}{2}$, respectively. When $\mu_{X} \leq \frac{1}{2}$, a comparison with Eq. (3) shows that the additional information $\mathrm{q} \leq \mathrm{p}$ does not sharpen the upper bound on $\beta$; however, for $\mu_{X}>\frac{1}{2}$ the upper bound is reduced to $\left(\sigma_{Y}^{2} / \sigma_{X Y}\right) \sqrt{1-4 \sigma_{X}^{2}\left(1-\rho_{X Y}^{2}\right)}$.

Finally, suppose that $\mathrm{p}=\mathrm{q}$. Misclassification is now symmetric: conditional on $\left(\mathrm{Z}_{\mathrm{i}}, \mathrm{Y}_{\mathrm{i}}\right)$ false positives and false negatives are equally likely. Under Assumption 2.2 and Assumption 2.3 the bounds for $\beta$ follow immediately from Lemma 3:

$\frac{\sigma_{X Y}}{\sigma_{X}^{2}} \leq \beta \leq \frac{\sigma_{Y}^{2}}{\sigma_{X Y}} \sqrt{1-4 \sigma_{X}^{2}\left(1-\rho_{X Y}^{2}\right)}$.

If, in addition, $\mathrm{U}_{\mathrm{i}}$ is homoscedastic, then $\beta$ is typically identified:

\section{Lemma 4.}

Suppose that Assumption 2.1, Assumption 2.2 and Assumption 2.3 hold with $\mathrm{p}=\mathrm{q}$ and $E\left(U_{i}^{2} \mid Z_{i}\right)=E\left(U_{i}^{2}\right)=\sigma_{U}^{2}$. Then Bis identified, except when $\mu_{X}=\frac{1}{2}$.

The identification result can be understood in terms of solving the system of equations in (2). Symmetry eliminates one of the unknowns, and homoscedasticity leads to two equations for $\sigma_{0}^{2}$ and $\sigma_{1}^{2}$, instead of a single one for $\sigma_{Y}^{2}$. This yields a system of 5 equations in 5 unknowns, which has a unique solution when $\mu_{X} \neq \frac{1}{2}$.

\section{Discussion}

In this paper we have analyzed partial identification of the regression coefficient of a binary misclassified variable. In particular, we have shown how various assumptions about the misclassification probabilities and the regression error variance affect the bounds of the identified set. Interestingly, these assumptions only affect the upper bound. In the case of Lemma 4 the regression parameter is identified. For most applications, however, the assumptions of homoscedasticity and symmetric misclassification are too strong. The identification strategies of Mahajan (2006) and Lewbel (2007), based on the availability of an instrumental variable, may then be more appropriate.

The use of conditional moments is common in identification analyses. Chen et al. (2008b) show that with homoscedasticity and the additional assumption that $E\left(U_{i}^{3} \mid Z_{i}\right)=E\left(U_{i}^{3}\right)$, the regression parameter is identified. We demonstrate here (Lemma 1) that dropping the restriction on the third moment results in partial identification. Our bounds are sharper, however, than those for the general heteroscedastic case in Bollinger (1996). On the other hand, Deng and Hu (2009) show that the identified set can be unbounded if the misclassification error is related to the outcome $\mathrm{Y}_{\mathrm{i}}$. The assumption of nondifferential measurement error therefore carries important identifying 
information, because it bounds the identified set. Additional information about the misclassification rates, if deemed plausible, can further sharpen the bounds (Lemma 2 and Lemma 3).

Appendix.

\section{Proof of Lemma 1.}

From Assumption 2.1 and Bayes' rule it follows that

$\operatorname{Pr}\left\{Z_{i}=1 \mid X_{i}=0\right\}=\frac{\pi q}{1-\mu_{X}}$,

$\operatorname{Pr}\left\{Z_{i}=1 \mid X_{i}=1\right\}=\frac{\pi(1-q)}{\mu_{X}}$.

The conditional variance of $\mathrm{Z}_{\mathrm{i}}$ given $\mathrm{X}_{\mathrm{i}}$ is

$$
\begin{gathered}
\mathrm{V}\left(Z_{i} \mid X_{i}=1\right)=\frac{\pi(1-\pi) p(1-q)}{\mu_{X}^{2}} . \\
\mathrm{V}\left(Z_{i} \mid X_{i}=1\right)=\frac{\pi(1-\pi) p(1-q)}{\mu_{X}^{2}} .
\end{gathered}
$$

Assumption 2.1 and homoscedasticity imply that

$$
\begin{aligned}
& \mathrm{E}\left(\mathrm{U}_{\mathrm{i}} \mid \mathrm{X}_{\mathrm{i}}\right)=\mathrm{E}\left[\mathrm{E}\left(\mathrm{U}_{\mathrm{i}} \mid \mathrm{Z}_{\mathrm{i}}, \mathrm{X}_{\mathrm{i}}\right) \mid \mathrm{X}_{\mathrm{i}}\right]=\mathrm{E}\left[\mathrm{E}\left(\mathrm{U}_{\mathrm{i}} \mid \mathrm{Z}_{\mathrm{i}}\right) \mid \mathrm{X}_{\mathrm{i}}\right]=0, \\
& \begin{aligned}
E\left(U_{i}^{2} \mid X_{i}\right) & =E\left[E\left(U_{i}^{2} \mid Z_{i}\right) \mid X_{i}\right] \\
& =\sigma_{U^{*}}^{2}
\end{aligned}
\end{aligned}
$$

The conditional variance of $\mathrm{Y}_{\mathrm{i}}$ given $\mathrm{X}_{\mathrm{i}}$ can now be calculated as

equation(A.1)

$\sigma_{0}^{2}=\beta^{2} \frac{\pi(1-\pi) q(1-p)}{\left(1-\mu_{X}\right)^{2}}+\sigma_{U}^{2}$,

equation(A.2)

$$
\sigma_{1}^{2}=\beta^{2} \frac{\pi(1-\pi)(1-q) p}{\mu_{X}^{2}}+\sigma_{U}^{2}
$$

From the first and third equations in (2) we can solve for $\beta$ as

equation(A.3)

$$
\begin{aligned}
\beta & =\frac{\sigma_{X Y}(1-p-q)}{\left(\mu_{X}-p\right)\left(1-\mu_{X}-q\right)} \\
& =b(p, q) .
\end{aligned}
$$


Substituting $\beta$ and $\pi=\left(\mu_{X}-p\right) /(1-p-q)$ in and , and using $\sigma_{U}^{2} \geq 0$, we obtain:

equation(A.4)

$0 \leq p \leq \mu_{X}\left[\frac{\mu_{X}^{2} \sigma_{1}^{2}\left(1-\mu_{X}-q\right)}{\mu_{X}^{2} \sigma_{1}^{2}\left(1-\mu_{X}-q\right)+\sigma_{X Y}^{2}(1-q)}\right]$,

equation(A.5)

$0 \leq q \leq\left(1-\mu_{X}\right)\left[\frac{\left(1-\mu_{X}\right)^{2} \sigma_{0}^{2}\left(\mu_{X}-p\right)}{\left(1-\mu_{X}\right)^{2} \sigma_{0}^{2}\left(\mu_{X}-p\right)+\sigma_{X Y}^{2}(1-p)}\right]$.

The upper (lower) bound of the identified set is the maximum (minimum) of b(p,q), subject to the restrictions in and. Since $\partial b / \partial p, \partial b / \partial q>0$ the lower bound for $\beta$ is attained $a p=q=0$. For the upper bound, suppose first that

$\left[\frac{\partial b(p, q)}{\partial p}\right]_{p=q=0}=\frac{\sigma_{X Y}}{\mu_{X}^{2}} \geq \frac{\sigma_{X Y}}{\left(1-\mu_{X}\right)^{2}}=\left[\frac{\partial b(p, q)}{\partial q}\right]_{p=q=0}$.

or $\mu_{X} \leq \frac{1}{2}$. Starting at $(0,0)$ it is optimal to increase p. Moreover, since $\mathrm{d}^{2} \mathrm{~b} / \mathrm{dp}^{2}>0$ for $\mathrm{q}=0$ and all feasible values of $p$, it is optimal to increase $p$ until the upper bound of (A.4) is binding. The first upper bound is then obtained by setting $\mathrm{q}=0$ and $p=\mu_{X}\left[\frac{\mu_{X}^{2} \sigma_{1}^{2}\left(1-\mu_{X}\right)}{\mu_{X}^{2} \sigma_{1}^{2}\left(1-\mu_{X}\right)+\sigma_{X Y}^{2}}\right]$,

in (A.3). A similar argument shows that for $\mu_{X}>\frac{1}{2}$ the second upper bound follows by substituting $\mathrm{p}=0$ and $p=\mu_{X}\left[\frac{\mu_{X}^{2} \sigma_{1}^{2}\left(1-\mu_{X}\right)}{\mu_{X}^{2} \sigma_{1}^{2}\left(1-\mu_{X}\right)+\sigma_{X Y}^{2}}\right]$,

into (A.3).

To show the second statement of Lemma 1, we first derive an expression for $\sigma_{Y}^{2}$. From Bayes' rule:

$$
\begin{gathered}
\begin{array}{c}
E\left(Z_{i} \mid X_{i}\right)=\operatorname{Pr}\left\{Z_{i}=1 \mid X_{i}=1\right\} X_{i}+\operatorname{Pr}\left\{Z_{i}=1 \mid X_{i}=0\right]\left(1-X_{i}\right) \\
=\frac{\pi q}{1-\mu_{X}}+\frac{X_{i} \pi\left(1-\mu_{X}-q\right)}{\mu_{X}\left(1-\mu_{X}\right)}
\end{array} \\
\mathrm{V}\left[E\left(Z_{i} \mid X_{i}\right)\right]=\frac{\pi^{2}\left(1-\mu_{X}-q\right)^{2}}{\mu_{X}\left(1-\mu_{X}\right)} .
\end{gathered}
$$

Using $\pi=\left(\mu_{X}-p\right) /(1-p-q)$ and Eq. (A.3), we get 


$$
\begin{aligned}
\sigma_{Y}^{2} & =E\left[\mathrm{~V}\left(Y_{i} \mid X_{i}\right)\right]+\mathrm{V}\left[E\left(Y_{i} \mid X_{i}\right)\right] \\
& =\mu_{X} \sigma_{1}^{2}+\left(1-\mu_{X}\right) \sigma_{0}^{2}+\mathrm{V}\left[\alpha+\beta E\left(Z_{i} \mid X_{i}\right)+E\left(U_{i} \mid X_{i}\right)\right] \\
& =\mu_{X} \sigma_{1}^{2}+\left(1-\mu_{X}\right) \sigma_{0}^{2}+\frac{\beta^{2} \pi^{2}\left(1-\mu_{X}-q\right)^{2}}{\mu_{X}\left(1-\mu_{X}\right)} \\
& =\mu_{X} \sigma_{1}^{2}+\left(1-\mu_{X}\right) \sigma_{0}^{2}+\frac{\sigma_{X Y}^{2}}{\sigma_{X}^{2}} .
\end{aligned}
$$

Suppose $\mu_{X} \leq \frac{1}{2}$, and consider the difference between the corresponding bounds in (3) and Lemma 1. Using the expression for $\sigma_{Y}^{2}$ given above:

$$
\begin{aligned}
\Delta_{1}= & \left(\mu_{X} \frac{\sigma_{X Y}}{\sigma_{X}^{2}}+\left(1-\mu_{X}\right) \frac{\sigma_{Y}^{2}}{\sigma_{X Y}}\right) \\
& -\left(\left(1-\mu_{X}\right) \frac{\sigma_{X Y}}{\sigma_{X}^{2}}+\mu_{X} \frac{\sigma_{1}^{2}\left(1-\mu_{X}\right)^{2}}{\sigma_{X Y}}\right) \\
= & \mu_{X} \frac{\sigma_{X Y}}{\sigma_{X}^{2}}+\left(1-\mu_{X}\right)\left(\mu_{X} \frac{\sigma_{1}^{2}}{\sigma_{X Y}}+\left(1-\mu_{X}\right) \frac{\sigma_{0}^{2}}{\sigma_{X Y}}+\frac{\sigma_{X Y}}{\sigma_{X}^{2}}\right) \\
& -\left(1-\mu_{X}\right) \frac{\sigma_{X Y}}{\sigma_{X}^{2}}-\mu_{X} \frac{\sigma_{1}^{2}\left(1-\mu_{X}\right)^{2}}{\sigma_{X Y}} \\
= & \mu_{X} \frac{\sigma_{X Y}}{\sigma_{X}^{2}}+\frac{1}{\sigma_{X Y}}\left[\sigma_{1}^{2} \mu_{X} \sigma_{X}^{2}+\sigma_{0}^{2}\left(1-\mu_{X}\right)^{2}\right] \\
> & 0 .
\end{aligned}
$$

Similarly, for $\mu_{X}>\frac{1}{2}$ the difference is

$$
\beta=\frac{\sigma_{X Y}(1-p)}{\left(\mu_{X}-p\right)\left(1-\mu_{X}\right)}=b(p)
$$

\section{Proof of Lemma 2.}

Suppose first that $q=0$, so that $\pi=\left(\mu_{X}-p\right) /(1-p)$, and

$$
\beta=\frac{\sigma_{X Y}(1-p)}{\left(\mu_{X}-p\right)\left(1-\mu_{X}\right)}=b(p)
$$

We now need to minimize and maximize b(p), subject to constraints. From ${ }^{\sigma_{U}^{2}} \geq 0$ and the system (2), it follows that

$0 \leq p \leq \mu_{X}\left(1-\rho_{X Y}^{2}\right)$. 
Since $d b(p) / d p>0$, the maximum and minimum of $b(p)$ are attained at $\mu_{X}\left(1-\rho_{X Y}^{2}\right)$ and 0 , respectively. Substituting these values in $b(p)$ yields the result. The argument for the case $p=0$ is completely analogous.

\section{Proof of Lemma 3.}

Consider b(p,q) in (A.3). Since b(p,q) is increasing in both p and q, the lower bound is obtained atp $=\mathrm{q}=0$, and $b(0,0)=\sigma_{X Y} / \sigma_{X}^{2}$. For the upper bound, first consider the case $\mu_{X} \leq \frac{1}{2}$.

Then $\partial b(p, q) / \partial p \geq \partial b(p, q) / \partial q>0$ at $(0,0)$, and it is optimal to increase $p$ until the nonnegativity of $\sigma_{U}^{2}$ is binding. This occurs at $p=\mu_{X}\left(1-\rho_{X Y}^{2}\right)$, and substitution of this into b(p,q) yields the first bound in Lemma 3. Now suppose that $\mu_{X}>\frac{1}{2}$, so that $0<\partial b(p, q) / \partial p<\partial b(p, q) / \partial q$ at the point $(0,0)$. It would be optimal to increase $q$, but now the constraint $q \leq p$ is binding. At the optimum we therefore must have $\mathrm{p}>0$. It is easy to show that $\sigma_{U}^{2}$ is zero when

equation(A.6)

$q=q(p)=\frac{\sigma_{X}^{2}\left(1-\rho_{X Y}^{2}\right)-p\left(1-\mu_{X}\right)}{\mu_{X}-p}$.

This curve intersects with the line $q=p$ at the point

equation(A.7)

$$
p^{*}=\frac{1}{2}-\frac{1}{2} \sqrt{1-4 \sigma_{X}^{2}\left(1-\rho_{X Y}^{2}\right)} \text {. }
$$

For maximizing $b(p, q)$ the constraint $q=p$ is binding when $p<p^{*}$, whereas (A.6) is binding for $\mathrm{p}>\mathrm{p}^{*}$. It remains to determine at which point the maximum can be found. Suppose that (A.6) holds at the solution. Substituting this into $b(p, q)$ and taking the derivative it follows that

$$
\frac{\partial b(p, q(p))}{\partial p}=-\frac{\sigma_{Y}^{2}}{\sigma_{X Y}}-\frac{\sigma_{X Y} \sigma_{X}^{2}\left(1-\rho_{X Y}^{2}\right)}{\rho_{X Y}^{2}\left(\mu_{X}-p\right)^{2}}<0 .
$$

Therefore, the value of $b(p, q)$ can be increased by decreasing $p$ to the point $p^{*}$, where also $\mathrm{q}=\mathrm{p}^{*}$ (note that a point (q,p*) with $\mathrm{q}<\mathrm{p}^{*}$ is not optimal, since $\mathrm{b}(\mathrm{p}, \mathrm{q})$ can be increased by increasing $q$ ). Substituting $q=p^{*}$ and (A.7) into the expression for $b(p, q)$ yields the second bound. The proof for the case $\mathrm{p} \leq \mathrm{q}$ is analogous and omitted here.

\section{Proof of Lemma 4.}

Assume first that $\mu_{X} \neq \frac{1}{2}$. Following the proof of Lemma 1 it can be shown that

$$
\sigma_{0}^{2}=\frac{\beta^{2} \pi(1-\pi) p(1-p)}{\left(1-\mu_{X}\right)^{2}}+\sigma_{U}^{2}
$$


$\sigma_{1}^{2}=\frac{\beta^{2} \pi(1-\pi) p(1-p)}{\mu_{X}^{2}}+\sigma_{U}^{2}$

Taking the difference of these two equations eliminates the structural parameter $\sigma_{U}^{2}$. Substituting $\pi=\left(\mu_{X}-p\right) /(1-2 p)$ and $b(p, q)$ with $\mathrm{p}=\mathrm{q}$ into the difference $\sigma_{1}^{2}-\sigma_{0}^{2}$, we find

$p=\frac{1}{2}-\frac{1}{2} \sqrt{1-4 \frac{c}{(1+c)} \sigma_{X}^{2}}$,

$c \equiv \frac{\left(\sigma_{1}^{2}-\sigma_{0}^{2}\right) \sigma_{X}^{4}}{\sigma_{X Y}^{2}\left(1-2 \mu_{X}\right)}$

Substituting the solution for p back into (A.3) we can solve for the coefficient as

$\beta=\frac{\sigma_{X Y}}{\sigma_{X}^{2}}(1+c) \sqrt{1-4 \frac{c}{(1+c)} \sigma_{X}^{2}}$.

When $\mu_{X}=\pi=\frac{1}{2}$ the conditional variance of $Y_{\mathrm{i}}$ does not depend on $\mathrm{X}_{\mathrm{i}}$ and we can no longer eliminate $\sigma_{U}^{2}$. The remaining parameters $\left(\alpha, \beta, \sigma_{U}^{2}, p\right)$ are now no longer identified.

\section{References}

Bollinger, C.R., 1996. Bounding mean regressions when a binary regressor is mismeasured. Journal of Econometrics 73, 387-399.

Carroll, R., Ruppert, D., Stefanski, L., 1995. Measurement Error in Nonlinear Models. Chapman \& Hall, London.

Chen, X., Hu, Y., Lewbel, A., 2008a. Nonparametric identification of regression models containing a misclassified dichotomous regressor without instruments. Economics Letters 100, 381-384.

Chen, X., Hu, Y., Lewbel, A., 2008b. A note on the closed-form identification of regression models with a mismeasured binary regressor. Statistics and Probability Letters 78, 1473-1479.

Deng, P., Hu, Y., 2009. Bounding the effect of a dichotomous regressor with arbitrary measurement errors. Economics Letters 105, 256-260.

Klepper, S., 1988. Bounding the effects of measurement error in regressions involving dichotomous variables. Journal of Econometrics 37, 343-359.

Kreider, B., Pepper, J.V., 2007. Disability and employment: reevaluating the evidence in light of reporting errors. Journal of the American Statistical Association 102, 432-441.

Lewbel, A., 2007. Estimation of average treatment effects with misclassification. Econometrica 75, 537-551. 
Mahajan, A., 2006. Identification and estimation of regression models with misclassification. Econometrica 74, 631-666.

Manski, C.F., 1990. Nonparametric bounds on treatment effects. American Economic Review 80, 319-323. 